\title{
Early Observations and Modern Ephemerides
}

\author{
E.M. Standish Jr \\ Caltech/JPL, Pasadena, U.S.A.
}

\begin{abstract}
There is a great variety of planetary and lunar observations recorded throughout history. Even today, some of these are useful for the improvement of ephemerides, though one must use a lot of judgment and caution - for surprising reasons. Five different sets of observations are presented, each with a story and a lesson: Galileo's observations of Neptune, Lalande's observations of Neptune, Williams' measurements of the 1780 solar eclipse, Robertson's 1811 transit timings, and solar eclipses in general.
\end{abstract}

\section{Introduction}

There are observations taken in the centuries past which are possibly useful for modern planetary ephemerides. Usually, but not always, these are observations of the outermost planets of the solar system, for those bodies have long periods - not fully covered by existing "modern" observations.

This paper discusses four different sets of observations, and each set presents a unique feature or two, interesting to investigate:

- the observation of Neptune by Galileo in 1613, which has possible astrometric value though there has been disagreement about its interpretation;

- the two observations of Neptune by Lalande in 1795, whose residuals are remarkably similar to each other;

- the observations of Uranus by Robertson in 1811 , where it is evident that at least some of the observations were fabricated; and

- the eclipse observations by Williams in 1780 , where the expedition missed totality but nevertheless obtained observations which seem to contain usable information.

\section{Galileo}

During the years 1610-1613, the astronomer Galileo discovered the four largest moons of Jupiter, charting their nightly positions, and finding that he was able to predict their future positions with a high degree of accuracy. Intriguingly, just when his measuring accuracy reached a peak, the planet Neptune passed close to, and indeed was occulted by, Jupiter. In 1979 Stillman Drake and Charles Kowal looked for and found the startling fact that in 1612-13 Galileo actually 
observed Neptune; not once, but three times -234 years before the "official" discovery.

One of the three observations is even more remarkable. On January 28, 1613 , Galileo drew a straight line from Jupiter which passed through two "fixed stars", and wrote, "After fixed star $a$ another followed in the same line thus; that is $b$, which also was observed on the preceding night; but they seemed farther apart then." One can find star $a$ in a star catalogue; one cannot find star $b$ : it is Neptune. So, it is unquestioned that Galileo saw Neptune, recorded its position in his notebook, and even made a comment about its motion.

Modern ephemerides predict that Neptune will be on the straight line connecting Jupiter and star $a$, within reasonably expected accuracy; however, the distance of Neptune from star $a$ does not agree with Galileo's drawing. The difference, distance $a-b$, deduced from Galileo's drawing versus that given by the ephemerides and star catalogues, corresponds to about 53 seconds of arc in Neptune's orbital longitude and, more importantly, about 26 seconds of arc in latitude. This disagreement is completely unacceptable for a modern ephemeris of Neptune.

There are two possible explanations:

1. there is something drastically wrong with the ephemeris of Neptune (as Drake and Kowal suggest), or

2. Galileo did not draw the star separation to scale, (he was not very interested in background stars).

For a full account of this story, see Drake \& Kowal (1980), Kowal \& Drake (1980), and Standish \& Nobili (1997).

\section{Lalande}

In 1795, the astronomer Michel Lalande recorded transit timings and circle (altitude) readings into his observing notebooks. It has long been known that on the evenings of May 8 and May 10, Lalande, unknowingly, measured the planet Neptune, thinking it to be a star. What is generally not known, however, is that someone noticed something strange about the observations. Michel's uncle, J.J. Lalande, later assembled his nephew's observations into the published Histoire Celeste. At some time, by someone, there was written in the margin of the notebook next to the May 8th measurement of the star preceeding Neptune, "see the 10th of May... there is a transposition of altitude and error on the passage of the other ... of the following star". And in the margin next to the May 10th measurement of Neptune, is written, "see the 8th of May... there is a transposition of altitude with the star which is at 595440 ". Whoever it was, he noticed something unusual, but he evidently assumed it to be a mere recording mixup. In fact, the order of transit between Neptune and the mentioned star had indeed changed over the two nights. If only...

Here, then, are two observations of the planet Neptune, taken 51 years before its discovery. With respect to a modern ephemeris, both right ascension residuals are nearly $-8^{\prime \prime}$ in right ascension and both are effectively 0 in declination, with uncertainties of about $4^{\prime \prime}$ in both components. Can these two observations be accommodated by modern ephemerides? No. Solving for ephemeris 
corrections fit to Lalande's observations as well as to the full set of observational measurements of Neptune shows clearly that Lalande's observations are not consistent with the other observations of Neptune.

A possible explanation is that Neptune would have appeared different from a star in Lalande's telescope - just enough ghost-like to cause an observer to hesitate long enough. Or, perhaps the two being so similar is just a coincidence. Without an explanation, these observations would be disconcerting in their implication.

For more detail on Lalande's observations, see Standish (1993).

\section{Robertson}

Evidently, there are many tens of thousands of transit timings and zenith distance measurements from Radcliffe Observatory in Oxford, covering the years 1811-1839, which have never been reduced. Those taken by Abram Robertson in 1811-12 show timings at five wires: sometimes listed to an integer number of seconds of time; sometimes listed to a half-second of time ("xx.5"), and even sometimes listed to a tenth of a second of time (" $x \mathrm{x} . \mathrm{x}$ "). However, closer scrutiny shows that starting on January 7, 1811 and continuing for a full month, the sum of the 1st and 5th wire readings (modulo 60) is always exactly equal to the sum of the 2nd and 4th readings, and these are both always exactly equal to twice the reading of the $3 \mathrm{rd}$ wire. Occasionally, a wire is recorded to one-quarter of a second; in all such cases, the opposite wire is recorded to three-quarters of a second, so that their sum is an integer (and always exactly equal to the other sums).

Can it possibly be that Robertson's observations are accurate to the level of 0.1 seconds? Not at all: he observed many of the same stars night after night, and the rms for any given observation is what should be expected for that time: well over half a second of time.

It is not known why many of the observations of Robertson were fabricated. For more detail, see Standish (1993) and, especially, Standish (1997).

\section{Williams}

In 1780 , an eclipse expedition was led by the Reverend Samuel Williams, sailing from Boston Harbor up the coast of Maine to Penobscot Bay. This was during the American Revolution, and the British were in control of the bay. After getting permission from the British to even enter the bay, after setting up the equipment at the observing site, and after taking measurements for a full week preceding the eclipse, the disastrous truth became known: the chosen site was outside the path of totality; they missed the eclipse!

Much has been conjectured and argued about the reason for the mistake; the competence and even the integrity of the leader has been discussed in a series of articles, referenced in Standish (1999).

Also described are some seemingly valid observations made during the approach and the recession of the eclipse: measurements of the "lucid part of the sun", the width of the partially exposed crescent of the Sun. Fitting to these 
measurements involves the adjustment of a priori values of the instrumental parameters (clock offset and rate, micrometer offset and scale), as well as for the astronomical factors (lunar longitude and latitude, earth rotation, site location). Despite all of the mystery surrounding this expedition, it seems that the observations are legitimate and can be comfortably fit with fairly reasonable adjustments to the relevant parameters. For a more detailed description, see Standish (1999).

\section{Conclusions}

Four sets of observations: the first two were taken by competent, legitimate observers; the second two were taken under rather suspicious circumstances.

At best, Galileo's measurement of Neptune can be used as a one-dimensional observation: Neptune was observed to be on the Jupiter-star $a$ line. For both Gaileo and Lalande, however, if one assumes that the Solar System model in the ephemerides is not complete, then it would be a mistake to use them; a present-era ephemeris would be best if fit to only present-era observations.

On the other hand, it seems that the non-fabricated observations of Robertson are legitimate; similarly, Williams' observations probably contain legitimate information about the orientation of the Earth at that time.

Ironically, then, it seems that the first two sets can not be used in presentday ephemeris adjustments, while the second two contain some useful information.

\section{References}

Drake, S., \& Kowal, C.T. 1980, "Galileo's Sighting of Neptune", Scientific American, 243, 74-81

Kowal, C.T., \& Drake, S. 1980, "Galileo's Observations of Neptune", Nature 287, 311-313

Standish, E.M. 1993, "Meridian Circle Observations of the Planets", in Galactic and Solar System Optical Astrometry, eds Morrison and Gilmore (Cambridge: Cambridge University Press)

Standish, E.M. 1997, "Fabricated Transit Data by Abram Robertson", DIO, 7.1, 3-13

Standish, E.M., \& Nobili, A.M. 1997, "Galileo's Observations of Neptune", Baltic Astronomy, 6, 99-106

Standish, E.M. 1999, "The Eclipse of 1780: A Revisit", in Anni Mirabiles: a Symposium Celebrating the 90th Birthday of Dorrit Hoffleit, eds Philip, van Altena, Upgren, eds (Schenectady, NY: L. Davis Press, Inc.) 